\title{
E-UNITARY INVERSE SEMIGROUPS OVER SEMILATTICES
}

\author{
by D. B. MCALISTER $†$
}

(Received 18 December, 1975)

1. Introduction. An inverse semigroup is called $E$-unitary if the equations $e a=e=$ $e^{2}$ together imply $a^{2}=a$. In a previous paper [4], the author showed that any $E$-unitary inverse semigroup is isomorphic to a semigroup constructed from a triple $(G, \mathscr{X}, \mathscr{Y})$ consisting of a down-directed partially ordered set $\mathscr{X}$, an ideal and subsemilattice $\mathscr{Y}$ of $\mathscr{Z}$ and a group $G$ acting on $\mathscr{X}$, on the left, by order automorphisms in such a way that $\mathscr{X}=G \mathscr{Y}$. This semigroup is denoted by $P(G, \mathscr{X}, \mathscr{Y})$; it consists of all pairs $(a, g) \in \mathscr{Y} \times G$ such that $\mathrm{g}^{-1} a \in \mathscr{Y}$, under the multiplication

$$
(a, g)(b, h)=(a \wedge g b, g h) .
$$

The aim of this paper is to give necessary and sufficient conditions on an inverse semigroup in order that it should be isomorphic to some $P(G, \mathscr{X}, \mathscr{Y})$ with $\mathscr{X}$ a semilattice. As well, we consider those congruences $\rho$ on an inverse semigroup $P(G, \mathscr{X}, \mathscr{Y})$ for which the quotient has the form $P(H, \mathcal{U}, \mathscr{V})$ for some triple $H, \mathscr{U}, \mathscr{V}$ as above, with $U$ a semilattice.

We shall assume familiarity with the construction and properties of $P(G, \mathscr{X}, \mathscr{Y})$ from [3], [4]. Undefined notation and terminology is that of Clifford and Preston [1]. In particular, when we are considering a partial order on an inverse semigroup, the partial order being referred to is the natural partial order; it is defined by

$$
a \leq b \text { if and only if } a=e b \text { for some } e^{2}=e \in S .
$$

Throughout the paper, when the terminology "triple $(G, \mathscr{X}, \mathscr{Y})$ " is used, it means that $\mathscr{Z}$ is a down-directed partially ordered set with $\mathscr{Y}$ an ideal and subsemilattice of $\mathscr{X}$, and that $G$ is a group acting on $\mathscr{X}$ by order automorphisms in such a way that $\mathscr{X}=G \mathscr{Y}$.

DefinITION 1.1. Let $S$ be an inverse semigroup. Then we say that $S$ is an $E$-unitary inverse semigroup over a semilattice if $S \cong P(G, \mathscr{X}, \mathscr{Y})$ for some triple $(G, \mathscr{X}, \mathscr{Y})$ with $\mathscr{X}$ a semilattice.

In terms of Definition 1.1, the aim of this paper is therefore to characterize $E$-unitary inverse semigroups over a semilattice.

\section{The general case}

Defintrion 2.1. Let $S$ be a partially ordered set and let $\theta: S \rightarrow T$ be a mapping of $S$ into a set $T$. Then $\theta$ is an $m$-map if, for each $t \in T$, the set $\{s \in S: s \theta=t\}$ has a maximum member.

† This research was partially supported by NSF Grant GP 27917. 
Let $S$ be an inverse semigroup. Then Munn [7] has shown that the relation $\sigma$ on $S$ defined by

$$
(a, b) \in \sigma \text { if and only if } e a=e b \text { for some } e^{2}=e \in S
$$

is the smallest congruence $\rho$ on $S$ for which $S / \rho$ is a group.

The following results about $\sigma$ will be used without comment in several places in the remainder of the paper.

LEMMA 2.2. (A) Let $S$ be an inverse semigroup and let $e, f$ be idempotents in $S$. Let $\sigma$ be the minimum group congruence on $S$. Then

$$
\sigma_{e, f}=\sigma \cap(e S f \times e S f)
$$

is the minimum group congruence on eSf. Similarly

$$
\sigma_{e}=\sigma \cap(S e \times S e)
$$

is the minimum group congruence on Se.

I.

(B) If $I$ is a non-empty ideal of $S$ then $\sigma \cap(I \times I)$ is the minimum group congruence on

(C) Let $(G, \mathscr{X}, \mathscr{Y})$ be a triple and let $S$ be an inverse subsemigroup of $P(G, \mathscr{X}, \mathscr{Y})$. Let $a=(u, g), b=(v, h)$ belong to $S$. Then

$$
(a, b) \in \sigma \text { if and only if } g=h \text {. }
$$

Proof. (A) Let $\gamma_{e, f}$ denote the restriction of $\gamma=\sigma^{\natural}$ to a homomorphism of eSf into $G=S / \sigma$. Then, since for each $a \in S$,

$$
a \gamma=e \gamma a \gamma f \gamma=(e a f) \gamma
$$

because $e \gamma=f \gamma=1$ (the identity of $G$ ), $\gamma_{e, f}$ is a homomorphism of $e S f$ onto $G$. Thus $\sigma_{e, f}$ is a group congruence on $e S f$.

On the other hand, suppose that $\rho$ is a group congruence on $e S f$ and let $(a, b) \in \sigma_{e, f}$. Then $a u=b u$ for some idempotent $u \in S$. This implies

$$
a(e u f)=(a u) e f=(b u) e f=b(e u f)
$$

since idempotents commute. But, since euf $\epsilon e S f$ and $\rho$ is a group congruence on eSf, it follows from these equalities that $(a, b) \in \rho$. Hence $\sigma_{e, f} \subseteq \rho$. In the same way it can be shown that $\sigma_{e}$ is the minimum group congruence on $S e$.

(B) The proof of this is similar.

(C) Suppose that $(a, b) \in \sigma$. Then $a e=b e$ for some idempotent $e=(f, 1) \in S$; thus $g=h$. On the other hand, suppose that $g=h$. Let $e=b^{-1} a a^{-1} b \in S$. Then $a e=b e$ and so $(a, b) \in \sigma$.

Let $S$ be an inverse semigroup and let $e, f$ be idempotents of $S$. Then we shall follow the notation introduced in Lemma 2.2 and denote by $\gamma_{e, f}$ the restriction of $\sigma^{\natural}$ to eSf and by $\gamma_{e}$ the restriction of $\sigma^{\natural}$ to $S e$; each is a homomorphism onto $G=S / \sigma$.

THEOREM 2.3. Let $(G, \mathscr{X}, \mathscr{Y})$ be a triple and set $S=P(G, \mathscr{X}, \mathscr{Y})$. Then $\mathscr{X}$ is a semilattice if and only if, for each pair of idempotents $e, f \in S, \gamma_{e, f}: e S f \rightarrow S / \sigma$ is an m-map. 
Proof. Since $\mathscr{X}=G \mathscr{Y}$ and $G$ acts by order automorphisms, it is easy to see that $\mathscr{X}$ is a semilattice if and only if $a \wedge e$ exists for each $a \in \mathscr{X}, e \in \mathscr{Y}$.

Suppose that $\mathscr{X}$ is a semilattice. Let $e=(u, 1), f=(v, 1)$ with $u, v \in \mathscr{Y}$, and pick $g \in G$. Then $s \in e S f$ is such that $s \sigma^{\natural}=g$ if and only if $s=(b, g)$ for some $b \leq u$ with $g^{-1} b \leq v$. If this is the case, then $b \leq u, g v$ and, by hypothesis, $u \wedge g v$ exists. Hence $b \leq u \wedge g v$ so that $(b, g) \leq(u \wedge g v, g) \in e S f ;$ but $(u \wedge g v, g) \sigma^{\natural}=g$. Thus

$$
(u \wedge g v, g)=\max \left\{s \in e S f: s \sigma^{\natural}=g\right\}
$$

and, since $g$ was arbitrarily chosen in $G, \gamma_{e, f}$ is consequently an $m$-map.

Conversely, suppose that each $\gamma_{e, f}$ is an $m$-map and let $a \in \mathscr{X}, e \in \mathscr{Y}$; then $a=g f$ for some $g \in G, f \in \mathcal{Y}$. By hypothesis, the set

$$
\left\{s \in(e, 1) S(f, 1): s \sigma^{\natural}=g\right\}
$$

has a maximum member $(c, g)$. Since $(c, g) \in(e, 1) S(f, 1), c \leq e, g^{-1} c \leq f$, so that $c \leq e, a$. On the other hand, if $b \leq e$, a then $(b, g) \in(e, 1) S(f, 1)$. But $(b, g) \sigma^{\natural}=g$, so that $(b, g) \leq(c, g)$; that is $b \leq c$. Hence $c=e \wedge a$ exists and $\mathscr{X}$ is consequently a semilattice.

Corollary 2.4. An inverse semigroup $S$ is an E-unitary inverse semigroup over a semilattice if and only if $S$ is E-unitary and each $\gamma_{e, f}$ is an $m$-map.

DefinItion 2.5 [6]. An inverse semigroup $S$ is $F$-inverse if and only if $\sigma^{\natural}: S \rightarrow S / \sigma$ is an $m$-map.

McFadden and O'Carroll [6] showed that an $F$-inverse semigroup has an identity. On the other hand, it is shown in [4] that an inverse monoid is $E$-unitary over a semilattice if and only if it is $F$-inverse. This result is expressed in the context of this paper by the next theorem.

THEOREM 2.6. Let $S$ be an inverse semigroup. Then the following statements are equivalent:

(i) $S$ is F-inverse;

(ii) $S$ has an identity and each $\gamma_{\text {e.f }}: e S f \rightarrow S / \sigma$ is an $m$-map;

(iii) $S \cong P(G, \mathscr{X}, \mathscr{Y})$ for some triple $(G, \mathscr{X}, \mathscr{Y})$ with $\mathscr{X}$ a semilattice and $\mathscr{Y}$ a principal ideal of $\mathscr{X}$.

Proof. (i) $\Rightarrow$ (ii). As pointed out above, McFadden and O'Carroll [6] have shown that any $F$-inverse semigroup has an identity; the identity is the element $e=$ $\max \left\{s \in S: s \sigma^{\natural}=1\right\}$, where 1 denotes the identity of $S / \sigma$.

Let $u, v$ be idempotents of $S$ and, for $g \in G$, let $h=\max \left\{s \in S: s \sigma^{\natural}=g\right\}$. Then $u h v \in u S v$ and $(u h v) \sigma^{\natural}=g$. If $s \in u S v$ is such that $s \sigma^{\natural}=g$ then $s \leq h$ and so $s=u s v \leq u h v$. Hence $u h v=\max \left\{s \in u S v: s \sigma^{\natural}=g\right\}$; it follows that $\gamma_{u, v}$ is an $m$-map.

(ii) $\Rightarrow$ (iii). Since $S$ has an identity, it follows from Corollary 2.4 that we need only verify that $S$ is $E$-unitary. Suppose that $f a=f=f^{2}$ for some $a \in S$. Then $a \sigma^{\natural}=1$ so that $a \leq \max \left\{s \in S: s \sigma_{e, e}=1\right\}$, where $e$ is the identity of $S$. But $e$ is a maximal element of $S=e S e$ so that $e=\max \left\{s \in S: s \sigma^{\natural}=1\right\}$; thus $a \leq e$. This implies $a=a a^{-1} e=a a^{-1}$, so that $a$ is idempotent. Hence $S$ is $E$-unitary. 
(iii) $\Rightarrow$ (i). Suppose $S=P(G, \mathscr{X}, \mathscr{Y})$ with $\mathscr{Z}$ a semilattice and $\mathscr{Y}$ a principal ideal of $\mathscr{Z}$, and let $e$ be the maximum element of $\mathscr{Y}$. Then, as in the proof of Theorem 2.3, $(e \wedge g e, g)$ is the maximum element $s$ of $S$ with $s \sigma^{\natural}=g$. Hence, $S$ is $F$-inverse.

Theorem 2.6 shows that, in the presence of an identity, the condition each $\gamma_{e, f}$ is an $m$-map ensures that $S$ is $E$-unitary. This is not the case in general.

EXAMPLE 2.7. Let $M_{2}$ be the Brandt semigroup $\mathscr{M}^{\circ}(\{1\},\{1,2\},\{1,2\}, \Delta)$. Then $M_{2}$ has the multiplication table

\begin{tabular}{c|ccccc} 
& 0 & $a$ & $a^{-1}$ & $e$ & $f$ \\
\hline 0 & 0 & 0 & 0 & 0 & 0 \\
$a$ & 0 & 0 & $e$ & 0 & $a$ \\
$a^{-1}$ & 0 & $f$ & 0 & $a^{-1}$ & 0 \\
$e$ & 0 & $a$ & 0 & $e$ & 0 \\
$f$ & 0 & 0 & $a^{-1}$ & 0 & $f$
\end{tabular}

with $a=(1,2), a^{-1}=(2,1), e=(1,1), f=(2,2)$.

In $M_{2}, e S e=\{e, 0\}, e S f=\{a, 0\}, f S e=\left\{a^{-1}, 0\right\}, f S f=\{f, 0\}$ and all other $u S v$ with $u^{2}=u$, $v^{2}=v$ are $\{0\}$. Hence each $\gamma_{u, v}$ is an $m$-map. But $S=M_{2}$ is not $E$-unitary.

In a sense, $M_{2}$ is the only counterexample to the hypothesis:

if each $\gamma_{e, f}$ is an $m$-map, then $S$ is $E$-unitary.

Before verifying this, we prove a lemma.

DefINTIION 2.8 [9]. Let $S$ be an inverse semigroup. Then $S$ is $E$-reflexive if and only if, for $a, b \in S, a b$ is idempotent if and only if $b a$ is idempotent.

LEMMA 2.9. Let $S$ be an inverse semigroup. Then the following statements are equivalent:

(i) there exists $a \in S$ such that $a^{2}<a$;

(ii) $S$ contains an isomorphic copy of $M_{2}$;

(iii) $S$ is not E-reflexive.

Proof. (i) $\Rightarrow$ (ii). Suppose $a^{2}<a$. Then $a^{2}=a^{2} a^{-2} a$ so that $a^{3}=a^{2} a^{-2} a \cdot a=a^{2}$. This implies that $a^{2}$ is idempotent and $a^{2}=a^{-2}$. Consider the subsemigroup $T$ of $S$ generated by $a$ and $a^{-1}$. Then, since $T$ is a homomorphic image of the free inverse semigroup on one generator, it follows from [2] that each element of $T$ is of the form $a^{r} a^{-s} a^{t}$ with $r$, $t \leq s$. Because $a^{2}=a^{3}=a^{-3}=a^{-2}$, one sees that $T$ has at most five members, $a, a^{-1}$, $e=a a^{-1}, f=a^{-1} a$ and $0=a^{2}$. Indeed, all five are distinct since, otherwise, $a$ would belong to some subgroup of $S$ and this would contradict $a^{2}<a$. Thus $T$ has the multiplication table in Example 2.7. That is, $T \cong M_{2}$.

(ii) $\Rightarrow$ (iii). Suppose $M_{2} \subseteq S$ and let $a=(1,2), b=(1,1)$. Then $a b=0$ is idempotent but $b a=a$ is not.

(iii) $\Rightarrow$ (i) Let $c, d \in S$ be such that $c d$ is idempotent but $a=d c$ is not. Then $a^{2}=a^{3}$ so that $a^{2}<a$. 
ThEOREM 2.10. Let $S$ be an inverse semigroup. Then $S$ is an E-unitary inverse semigroup over a semilattice if and only if $S$ is $E$-reflexive and each $\gamma_{e, f}: e S f \rightarrow S / \sigma$ is an m-map.

Proof. Suppose that $S$ is $E$-reflexive and that each $\gamma_{e, f}$ is an $m$-map. Let $e a=e=e^{2}$. Then, since $a$ is a maximal member of $a a^{-1} S a^{-1} a$,

$$
a=\max \left\{b \in a a^{-1} S a^{-1} a: b \sigma^{\natural}=1\right\} .
$$

Thus $a^{2} \leq a$ so that, by Lemma $2.9, a^{2}=a$. Hence $S$ is $E$-unitary and so, by Corollary 2.4 , $S \cong P(G, \mathscr{X}, \mathscr{Y})$ where $\mathscr{X}$ is a semilattice.

Conversely, let $S \cong P(G, \mathscr{X}, \mathscr{Y})$, where $\mathscr{X}$ is a semilattice. Then, since $M_{2}$ is not $E$-unitary, $S$ does not contain $M_{2}$. Thus, by Lemma 2.9, $S$ is $E$-reflexive. Further, Theorem 2.3 shows that each $\gamma_{e, f}$ is an $m$-map.

It is an easy matter to see that if an inverse semigroup $T$ is $E$-reflexive and each $\gamma_{e, f}: e T f \rightarrow T / \sigma$ is an $m$-map, then the same is true for each ideal of $T$. In particular, if an inverse semigroup $S$ is embedded as an ideal in an $F$-inverse semigroup $T$ then $S$ is $E$-unitary over a semilattice. Example 2.13 shows that the converse need not be the case.

LEMMA 2.11. Let $(G, \mathcal{U}, \mathscr{V})$ be a triple and let $\mathscr{Y}$ be an ideal of $\mathscr{V}$, thus of $\mathscr{U}$, and set $\mathscr{X}=G \mathscr{Y}$. Suppose that $\mathscr{X} \cap \mathscr{V}=\mathscr{Y}$. Then $(G, \mathscr{X}, \mathscr{Y})$ is a triple and $P(G, \mathscr{X}, \mathscr{Y})$ is an ideal of $P(G, \mathscr{U}, \mathscr{V})$; if $\mathcal{U}$ is a semilattice, so is $\mathscr{Z}$.

Conversely, if $S$ is an ideal of $P(G, \mathcal{U}, \mathscr{V})$ then $\mathscr{Y}=\{a \in \mathscr{V}:(a, 1) \in S\}$ is an ideal of $\mathscr{V}$ such that $G \mathscr{Y} \cap \mathscr{V}=\mathscr{Y}$. Further, $S=P(G, G \mathcal{Y}, \mathscr{Y})$.

Proof. This is straightforward.

THEOREM 2.12. Let $S$ be an inverse semigroup. Then the following statements are equivalent:

(1) each $\gamma_{e}: S e \rightarrow S / \sigma$ is an $m-$ map, for $e^{2}=e \in S$;

(2) the translational hull $\Omega(S)$ of $S$ is F-inverse;

(3) $S$ can be embedded as an ideal in an F-inverse semigroup.

Proof. (1) $\Rightarrow(2)$. Suppose that (1) holds. We first show that $S$ is E-unitary. Suppose that $e a=e=e^{2}$. Then $a \in S a^{-1} a$ is such that $a \sigma^{\natural}=1$. By hypothesis, the set $\left\{s \in S a^{-1} a: s \sigma^{b}=1\right\}$ has a maximum member $t$; thus $a, a^{-1} a \leq t$. But $a, a^{-1} a$ are maximal in $S a^{-1} a$, from which it follows that $t=a=a^{-1} a$. Thus $a^{2}=a$ and so $S$ is $E$-unitary.

We may therefore suppose that $S=P(G, \mathscr{X}, \mathscr{Y})$ for some triple $(G, \mathscr{X}, \mathscr{Y})$. Let $\mathscr{X}^{*}$ and $\mathscr{Y}^{*}$ denote the set of all non-empty order ideals of $\mathscr{X}$ and $\mathscr{Y}$, respectively, under inclusion, and let $G$ act on $\mathscr{X}^{*}$ by $g A=\{g a: a \in A\}$ for each $A \in \mathscr{X}^{*}$. Let $\mathscr{Z}^{*}=G^{*}$. Then $\left(G, \mathscr{Z}^{*}, \mathscr{Y}^{*}\right)$ is a triple and we may regard $S$ as being embedded in $P\left(G, \mathscr{Z}^{*}, \mathscr{Y}^{*}\right)$ by $(a, g) \mapsto(\bar{a}, g)$, where, for $a \in \mathscr{X}, \bar{a}=\{x \in \mathscr{X}: x \leq a\}$. Assume that this has been done. Then it is shown in [5, Section 3] that $\Omega(S)$ is isomorphic to the idealizer of $S$ in $P\left(G, \mathscr{Z}^{*}, \mathscr{Y}^{*}\right)$. Further, it is shown there that $\hat{S}=P\left(G, \mathscr{Z}^{*}, \mathscr{Y}^{*}\right)$ is an $F$-inverse semigroup in which, for each $g \in G,\left(I_{g}, g\right)$ is the maximum element $t$ of $\hat{S}$ with $t \sigma^{\natural}=g$; here $I_{g}=\mathscr{Y} \cap g \mathscr{Y}$. 
It follows from these remarks and Lemma 2.2 that in order to show that $\Omega(S)$ is $F$-inverse, it suffices to show that $\left(I_{g}, g\right)$ is in $\Omega(S)$ for each $g \in G$. It is shown in [5, Theorem 3.9] that $\Omega(S)$ consists of all pairs $(A, g) \in P\left(G, \mathscr{Z}^{*}, \mathscr{Y}^{*}\right)$ such that, for each $e \in \mathscr{Y},\{x \in A: x \leq e\}$ has a maximum member.

Let $e \in \mathscr{Y}, g \in G$. Then, by hypothesis, the set $\left\{s \in S(e, 1): s \sigma^{\natural}=g^{-1}\right\}$ has a maximum member $t=\left(g^{-1} c, g^{-1}\right)$ for some $c \in \mathscr{Y}$. Since $t \in S(e, 1), t^{-1} t \leq(e, 1)$; that is, $c \leq e$. Hence $c \in\left\{x \in \mathscr{Y} \cap g^{\mathscr{Y}}: x \leq e\right\}$. On the other hand, if $x \in \mathscr{Y} \cap g^{\mathscr{Y}}, x \leq e$ then $\left(g^{-1} x, g^{-1}\right) \in S(e, 1)$ and $\left(g^{-1} x, g^{-1}\right) \sigma^{\natural}=g^{-1}$. This means that $\left(g^{-1} x, g^{-1}\right) \leq\left(g^{-1} c, g^{-1}\right)$ so that $g^{-1} x \leq g^{-1} c$ and so $x \leq c$. It follows that $c=\max \left\{x \in I_{g}: x \leq e\right\}$. Hence $\left(I_{g}, g\right) \in \Omega(S)$ and then $\left(I_{g}, g\right)=$ $\max \{s \in \Omega(S): s \sigma=g\}$. Consequently $\Omega(S)$ is $F$-inverse.

(2) $\Rightarrow(3)$ is immediate, since $S$ is an ideal of $\Omega(S)$.

$(3) \Rightarrow(1)$. Suppose that $S$ is an ideal of an $F$-inverse semigroup $T$. By Theorem 2.6 we may assume $T=P(G, \mathcal{U}, \mathscr{V})$, where $\mathscr{U}$ is a semilattice and $\mathscr{V}$ is a principal ideal with greatest element $v$. Let $e=(f, 1) \in S$ and pick $g \in G$. Then $g^{-1} v \wedge f \leq f$ so that $g^{-1} v \wedge f \in \mathscr{Y}$, where $\mathscr{Y}=\{u \in \mathscr{Q}:(u, 1) \in S\}$, and $g\left(g^{-1} v \wedge f\right) \geq v$ so that $g\left(g^{-1} v \wedge f\right) \in \mathscr{V} \cap G \mathscr{Y}=\mathscr{Y}$, by Lemma 2.11. Now by Lemma 2.11, $S=P(G, G \mathscr{Y}, \mathcal{Y})$. Hence $\left(g^{-1} v \wedge f, g^{-1}\right) \in S$ and so, consequently, $\left(g^{-1} v \wedge f, g^{-1}\right)^{-1}=(v \wedge g f, g) \in S$. Indeed, since $g^{-1}(g f \wedge v) \leq f,(v \wedge g f, g) \in S e$ and $(v \wedge g f, g) \sigma^{\natural}=g$.

On the other hand, suppose that $(a, g) \in S e$. Then $g^{-1} a \leq f$ and $a \in \mathscr{Y} \subseteq \mathscr{V}$ so that $a \leq v$. Hence $a \leq v \wedge g f$ and consequently $(a, g) \leq(v \wedge g f, g)$. It follows that $(v \wedge g f, g)=$ $\max \left\{s \in S e: s \sigma^{\natural}=g\right\}$; hence $\sigma_{e}$ is an $m$-map.

EXAMPLE 2.13. Let $\mathbb{Q}^{+}$be the set of positive rationals under the reverse of the usual ordering and let $Y=\left\{x \in \mathbb{Q}^{+}: x^{2}>2\right\}$. Then the group $G$ of positive rationals acts on $\mathbb{Q}^{+}$ by multiplication. Let $S=P\left(G, \mathbb{Q}^{+}, \mho\right)$ and let $e=(2,1) \in S, g=\frac{1}{3} \in G$. Then $(f, g) \in S e$ for all $f \in \mathbb{Y}$. Thus, as ordered sets,

$$
\left\{s \in S e: s \sigma^{\natural}=g\right\} \cong \mathbb{V} .
$$

But $\checkmark$ has no maximum member $\left(\sqrt{2}\right.$ is irrational) so that $\sigma_{e}$ is not an $m$-map. Hence $S$ cannot be embedded as an ideal in an $F$-inverse semigroup.

From the characterization of $\Omega(S)$ in [5, Theorem 3.9], one can show that $\Omega(S)=S^{1}$.

3. Some special cases. In this section, we consider some special cases in which it is possible to improve on the result in Theorem 2.10 .

Proposition 3.1. Let $S$ be an inverse semigroup and suppose that the semilattice of idempotents of $S$ is up-directed. Then $S$ is an E-unitary inverse semigroup over a semilattice if and only if eSe is F-inverse for each $e^{2}=e \in S$.

Proof. Suppose that $S$ is $E$-unitary over a semilattice and let $e^{2}=e \in S$. Then, by Theorem 2.3, $\gamma_{e, e}: e S e \rightarrow G=S / \sigma$ is an $m$-map. Since $e S e$ is an inverse semigroup, it follows, from Lemma 2.2(A), that $e S e$ is $F$-inverse. On the other hand, suppose that each $e S e$ is $F$-inverse. We show first that $S$ cannot contain $M_{2}$. Thus, by Lemma $2.9, S$ is $E$-reflexive. 
For any $a \in S$, there exists $e^{2}=e$ such that $e \geq a a^{-1}, a^{-1} a$. Then $a, a^{-1}$ belong to $e S e$ which is $E$-unitary, being $F$-inverse. Since $M_{2}$ is not $E$-unitary, it follows that $T=\left\langle a, a^{-1}\right\rangle$ is not isomorphic to $M_{2}$. Hence $S$ does not contain any copy of $M_{2}$.

Next, let $u, v$ be idempotents and let $e^{2}=e \geq u, v$. Then $u S v \subseteq e S e$ and, by hypothesis, $e S e$ is $F$-inverse. Let $g \in G=S / \sigma$. Then, as in the proof of Theorem 2.6,

$$
u\left(\max \left\{s \in e S e: s \sigma^{\natural}=g\right\}\right) v=\max \left\{s \in u S v: s \sigma^{\natural}=g\right\} .
$$

Hence $\gamma_{u, v}$ is an $m$-map for all idempotents $u, v \in S$.

It now follows from Theorem 2.10 that $S$ is $E$-unitary over a semilattice.

The results in the next two propositions are similar to Proposition 3.1. However, they depend on the algebraic structure of the semigroup $S$ rather than on the order structure of the idempotents of $S$.

Proposition 3.2. Let $S$ be an inverse semigroup which is a semilattice of groups. Then $S$ is an E-unitary inverse semigroup over a semilattice if and only if eSe is F-inverse for each $e^{2}=e \in S$.

Proof. As with Proposition 3.1, the condition is necessary. On the other hand, suppose that $e S e$ is $F$-inverse for each $e^{2}=e \in S$. Then, since $S$ cannot contain $M_{2}$, we need only show that each $\gamma_{u, v}$ is an $m$-map. But $u S v=u v S u v$ since idempotents are central, and then, by definition, $\sigma_{u, v}=\sigma_{u v, u v}$, so that the hypothesis in the statement of the proposition implies that each $\gamma_{u, v}$ is an $m$-map.

Proposition 3.3. Let $S$ be a simple E-unitary inverse semigroup. Then $S \cong P(G, \mathscr{X}, \mathscr{Y})$ for some triple $(G, \mathscr{X}, \mathscr{Y})$, with $\mathscr{X}$ a semilattice, if and only if, for some idempotent $e \in S$, eSe is F-inverse.

Proof. We need only show that the condition is sufficient. Suppose eSe is $F$-inverse for some idempotent $e$ and let $u, v$ be idempotents of $S$. Since $S$ is simple, there exist $b, c$ such that $b b^{-1}=u, b^{-1} b \leq e, c c^{-1}=v, c^{-1} c \leq e$. Suppose that $x \in u S v$ is such that $x \sigma^{\natural}=g \in$ $G$. Then $b^{-1} x c$ is in eSe since

$$
b^{-1} x c c^{-1} x^{-1} b \leq b^{-1} b \leq e \text { and } c^{-1} x^{-1} b b^{-1} x c \leq c^{-1} c \leq e .
$$

Further $\left(b^{-1} x c\right) \sigma^{\natural}=\left(b^{-1} \sigma^{\natural}\right) g\left(c \sigma^{\natural}\right)=h$, say, so that, by the hypothesis on eSe, $b^{-1} x c \leq z=$ $\max \left\{x \in e S e: x \sigma^{\natural}=h\right\}$. Then $x=b b^{-1} x c c^{-1} \leq b z c^{-1}$, and $b z c^{-1} \in u S v$ is such that $\left(b z c^{-1}\right) \sigma^{\natural}=$ g. Hence $b z c^{-1}=\max \left\{s \in u S v: s \sigma^{\natural}=g\right\}$, and so $\gamma_{u, v}$ is an $m$-map.

The requirement, in Proposition 3.3, that $S$ should be $E$-unitary is necessary as the following example shows.

EXAMPLE 3.4. Let $E$ be the $\omega$-tree with the Hasse diagram shown in Fig. 1 and let $S=T_{E}$ be the inverse semigroup of order isomorphisms between principal ideals of $E$. Then $E$ is uniform so that $S$ is bisimple.

For each $a \in E$, denote by $\varepsilon_{a}$ the identity mapping on the principal ideal $\bar{a}=$ $\{x \in E: x \leq a\}$. Then

$$
\varepsilon_{0} T_{E} \varepsilon_{0}=\left\{\alpha \in T_{E}: \Delta \alpha \cup \nabla \alpha \subseteq \overline{0}\right\}
$$




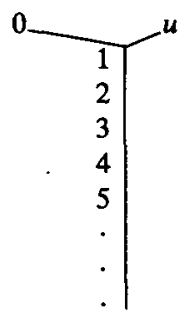

Figure 1

Thus $\varepsilon_{0} S \varepsilon_{0}$ is the semigroup of isomorphisms between principal ideals of $\{0,1, \ldots\}$. Consequently $\varepsilon_{0} S \varepsilon_{0} \cong B$, where $B$ is the bicyclic semigroup. McFadden and O'Carroll [6] have shown that $B$ is $F$-inverse.

On the other hand, let $\alpha \in T_{E}$ be defined by $\Delta \alpha=\overline{0}$ and

$$
x \alpha=\left\{\begin{array}{lll}
u & \text { if } & x=0 \\
x & \text { if } & x>0
\end{array}\right.
$$

Then $\alpha^{2}=\varepsilon_{1}<\alpha$ so that $S$ contains a copy of $M_{2}$. Hence $S$ is not $E$-unitary.

The last result is different in type from the earlier ones in this section since on this occasion we impose a restriction on the idempotents of $S$ and deduce a result about the maps $\gamma_{e, f}$.

Definition 3.5. A locally finite tree is a partially ordered set $X$ in which the following three conditions are satisfied:

(i) $X$ is down-directed;

(ii) if $a, b \leq c$ where $a, b, c \in X$, then $a \leq b$ or $b \leq a$;

(iii) the set $\{x \in X: a \leq x \leq b\}$ is finite for all $a, b \in X$ with $a \leq b$.

PROPOSITION 3.6. Let $S$ be an inverse semigroup whose idempotents form a locally finite tree. Then the following conditions on $S$ are equivalent:

(i) $S$ is E-unitary;

(ii) $S$ is E-unitary over a semilattice;

(iii) $\Omega(S)$ is $F$-inverse.

Proof. (i) $\Rightarrow$ (ii). Suppose that $S$ is $E$-unitary. Then $S \cong P(G, \mathscr{X}, \mathscr{Y})$ for some triple $(G, \mathscr{X}, \mathscr{Y})$, where $\mathscr{X}$ is down-directed and $G$ acts on $\mathscr{X}$ in such a way that $\mathscr{Z}=G \mathscr{Y}$. Since $P(G, \mathscr{L}, \mathscr{Y})$ has semilattice of idempotents $\mathscr{Y} \times 1, \mathscr{Y}$ is a locally finite tree. In fact, since $\mathscr{X}=G \mathscr{Y}$ and $\mathscr{X}$ is down-directed, it is easy to see that $\mathscr{X}$ is a locally finite tree. Hence $\mathscr{X}$ is a semilattice.

(ii) $\Rightarrow$ (iii). Again, we may suppose that $S=P(G, \mathscr{X}, \mathscr{Y})$ and, as in the proof of Theorem 2.12, it suffices to show that, for each $g \in G, e \in \mathcal{Y}$, the set $\left\{x \in I_{g}: x \leq e\right\}$ has a maximum member. 
For each $x \in I_{g}$ such that $x \leq e$, the set $\left\{y \in I_{g}: x \leq y \leq e\right\}$ has a maximum member $m_{x}$ since $\mathscr{Y}$ is locally finite. Fix such an $x$ and let $z \in I_{\mathrm{g}}$ with $z \leq e$. Then either $z \leq x$ or $x<z$. In the first case $z \leq x \leq m_{x}$ while, in the second, $x<z \leq e, z \in I_{g}$ imply $z \leq m_{x}$. Hence $m_{x}=\max \left\{y \in I_{g}: y \leq e\right\}$. This shows that $\left(I_{g}, g\right) \in \Omega(S)$, so that $\Omega(S)$ is $F$-inverse.

(iii) $\Rightarrow$ (i). This is immediate.

4. Congruences. In this section, we characterize those congruences on an $E$-unitary inverse semigroup over a semilattice whose quotient is also of this type.

DefINITION 4.1 [11]. Let $S$ be an inverse semigroup with semilattice of idempotents $E$. Then a normal partition on $E$ is an equivalence $\pi$ on $E$ such that

(i) if $e \pi f, u \in E$ then $e u \pi f u$;

(ii) if $e \pi f$ then $a^{-1} e a \pi a^{-1} f a$ for all $a \in S$.

If $\rho$ is a congruence on $S$ then $\pi_{\rho}=\rho \cap(E \times E)$ is a normal partition on $E$ and is called the normal partition induced by $\rho$.

If $\pi$ is a normal partition on $E$ then Reilly and Scheiblich [11] show that the smallest congruence $\rho_{\pi}$ on $S$ which induces $\pi$ is given by the following rule: $(a, b) \in \rho_{\pi}$ if and only if $e a=e b$ for some $e^{2}=e \in S$ such that $a a^{-1} \pi e \pi b b^{-1}$.

The result in the next lemma is due to Reilly and Munn [10]. They derived it by giving an explicit construction for $S / \rho_{\pi}$ in the form $P(G, \mathscr{X}, \mathscr{Y})$. We shall derive it as a direct consequence of Corollary 2.4 .

LEMMA 4.2. Let $S$ be an E-unitary inverse semigroup over a semilattice and let $\pi$ be a normal partition on the idempotents of $S$. Then $S / \rho_{\pi}$ is an $E$-unitary inverse semigroup over a semilattice.

Proof. We verify that the conditions of Corollary 2.4 hold for $T=S / \rho_{\pi}$. First, suppose that $(e, e a) \in \rho_{\pi}$ for some $e^{2}=e$. Then $f e=f e a$ for some idempotent $f$. Since $S$ is $E$-unitary, this implies that $a^{2}=a$. Hence $T$ is $E$-unitary.

Next, let $\bar{X}$ be a $\sigma$-class of $T$ and let $\bar{e}, \bar{f}$ be idempotents of $T$, say with $\bar{e}=e \rho_{\pi}$, $\bar{f}=f \rho_{\pi}, e^{2}=e, f^{2}=f$. Suppose $\bar{x} \in \bar{X} \cap \bar{e} T \bar{f}$ and let $x \in e S f$ be such that $x \rho_{\pi}=\bar{x}$. By hypothesis, $x \leq z$, where $z=\max \left\{s \in e S f: s \gamma_{e, f}=x \gamma_{e, f}\right\}$. Hence $\bar{x} \leq \bar{z}=z \rho_{\underline{\pi}}$. But, since $x \gamma_{e, f}=$ $z \gamma_{e, f}, u z=u x$ for some $u^{2}=u \in S$; thus $\bar{x} \sigma \bar{z}$. By the choice of $e, f, \bar{z} \in \bar{X} \cap \bar{e} T \bar{f}$. Hence $\bar{z}$ is the maximum element of $\bar{X} \cap \bar{e} T \bar{f}$. It follows that $\gamma_{\bar{e}, \bar{f}}$ is an $m$-map for each pair $\bar{e}, \bar{f}$ of idempotents of $T$. Therefore, by Corollary $2.4, T$ is an $E$-unitary inverse semigroup over a semilattice.

THEOREM 4.3 [10]. Let $S$ be an inverse semigroup. Then $S$ is an idempotent-separating homomorphic image of an E-unitary inverse semigroup over a semilattice.

Proof. Let $\theta: F I_{X} \rightarrow S$ be a homomorphism from a free inverse semigroup onto $S$. Then (cf. [8]) $F I_{X}^{1}$ is $F$-inverse and $F I_{X}$ is an ideal of $F I_{X}^{1}$. Hence, by Lemma $2.11, F I_{X}$ is $E$-unitary over a semilattice. 
Let $\pi=\theta \circ \theta^{-1} \cap(E \times E)$ where $E$ is the set of idempotents of $F I_{X}$. Then $\pi$ is a normal partition and $S$ is an idempotent-separating homomorphic image of $S / \rho_{\pi}$. The result now follows immediately from Lemma 4.2 .

The following corollary strengthens Theorem 4.2 of [4].

Corollary 4.4. Let $E$ be a semilattice. Then $E$ can be embedded as an ideal in a semilattice $F$ with the following property: each isomorphism between principal ideals of $F$ can be extended to an automorphism of $F$.

Proof. This follows from Theorem 4.3, using the argument in [4, Theorem 4.2].

We now turn to consider the idempotent-separating congruences on an inverse semigroup $P=P(G, \mathscr{X}, \mathscr{Y})$, where $\mathscr{X}$ is a semilattice. The next result is related to some of those in [10], and the proof is omitted.

Lemma 4.5. Let $(G, \mathscr{X}, \mathscr{Y})$ be a triple and let $N$ be a normal subgroup of $G$ such that $a \in \mathscr{Y}, n a \in \mathcal{Y}$ imply $a=n a$ for each $a \in \mathscr{Y}, n \in N$.

Then the relation $\rho_{N}$ defined by

$$
(a, g) \rho_{N}(b, h) \text { if and only if } a=b \text { and } g^{-1} h \in N
$$

is an idempotent-separating congruence on $S=P(G, \mathscr{X}, \mathscr{Y})$ such that $S / \rho_{N}$ is $E$-unitary.

Conversely, suppose that $\rho$ is an idempotent-separating congruence on $S$ such that $S / \rho$ is E-unitary. Then

$$
N=\{g \in G:(a, g) \rho(a, 1) \text { for some } a \in \mathscr{Y}\}
$$

is a normal subgroup of $G$ which satisfies condition (*). Further $\rho=\rho_{\mathrm{N}}$.

The next proposition gives necessary and sufficient conditions on $N$ in order that $T=S / \rho_{N}$ should be $E$-unitary over a semilattice. In the proof of the result, we shall denote the elements of $T$ by $[a, g]$ where $(a, g) \in S$. If $[a, g]=(a, g) \rho_{N}^{\natural} \in T$, it is easy to see that the mapping $[a, g] \rightarrow N g$ is a homomorphism of $T$ onto $G / N$ which induces $\sigma$. Hence we may identify $T / \sigma$ with $G / N$ by means of this mapping.

Propostrion 4.6. Let $S=P(G, \mathscr{X}, \mathscr{Y})$, where $\mathscr{L}$ is a semilattice, and let $N$ be a normal subgroup of $G$ which satisfies condition $(*)$. Then $T=S / \rho_{N}$ is $E$-unitary over a semilattice if and only if, for each $a, b \in \mathscr{X}$,

$$
\{a \wedge n b: n \in N\}
$$

has a maximum member.

Proof. First note that, by Lemma $4.5, T$ is $E$-unitary. Suppose that, for all $a, b \in \mathscr{L}$, $\{a \wedge n b: n \in N\}$ has a maximum member. Let $u, v$ be idempotents of $T$ and let $X=N g \in$ $T / \sigma$; then $u=[e, 1], v=[f, 1]$ for some $e, f \in \mathscr{Y}$. By hypothesis, $\bar{e}=\max \{e \wedge n g f: n \in N\}$ exists; say $\bar{e}=e \wedge n_{1} g f$. Then $\left[\bar{e}, n_{1} g\right] \in u T v$ and $\left[\bar{e}, n_{1} g\right] \sigma=N g$.

On the other hand, suppose that $[a, h] \in u T v$ is such that $[a, h] \sigma^{\natural}=N g$. Then, because $\rho_{N}$ is idempotent-separating, $a \leq e, h^{-1} a \leq f$ and, further, $h=n g$ for some $n \in N$. Thus 
$a \leq e \wedge n g f \leq \bar{e}$ so that $[a, h] \leq\left[\bar{e}, n_{1} g\right]$. Hence

$$
\left[\bar{e}, n_{1} g\right]=\max \left\{t \in u T v: t \sigma^{\natural}=N g\right\} .
$$

Thus, by Corollary $2.4, T$ is $E$-unitary over a semilattice.

Conversely, suppose that $T$ is $E$-unitary over a semilattice and let $a=g e, b=h f$, where $e, f \in \mathscr{Y}$ and $g, h \in G$. By the hypothesis that $T$ is over a semilattice,

$$
x=\max \left\{t \in[e, 1] T[f, 1]: t \sigma^{\natural}=N g^{-1} h\right\}
$$

exists. Then $x=[u, k]$, where $u \leq e, k^{-1} u \leq f$ and $N k=N g^{-1} h$; thus $k=g^{-1} n_{1} h$ and $u \leq e \wedge g^{-1} n_{1} h f$ for some $n_{1} \in N$, so that $g u \leq a \wedge n_{1} b$.

On the other hand, for any $n \in N, t=\left[e \wedge g^{-1} n h f, g^{-1} n h\right]$ is in $[e, 1] T[f, 1]$ and $t \sigma^{\natural}=N g^{-1} h$. Hence $t \leq x$ which, since $\rho_{N}$ is idempotent-separating, implies $e \wedge g^{-1} n h f \leq u$; that is, $a \wedge n b \leq g u$. But, as we have seen, $g u \leq a \wedge n_{1} b$, so we must have

$$
g u=a \wedge n_{1} b=\max \{a \wedge n b: n \in N\} .
$$

Corollary 4.7. Let $S=P(G, \mathscr{X}, \mathscr{Y})$, where $\mathscr{X}$ is a semilattice, and let $N$ be a normal subgroup of $G$ such that $n a=a$ for each $n \in N, a \in \mathscr{X}$. Then $S / \rho_{N}$ is $E$-unitary over a semilattice.

It was shown in [5] that every idempotent-separating congruence on $S=P(G, \mathscr{X}, \mathscr{Y})$, where $\mathscr{X}$ is a semilattice, can be extended to an idempotent-separating congruence on $P(G, \mathscr{X}, \mathscr{X})$. It is easy to see that $\rho_{N}$ can be extended to an $E$-unitary congruence if and only if $N \subseteq\{g \in G: g a=a$ for all $a \in \mathscr{X}\}$.

\section{REFERENCES}

1. A. H. Clifford and G. B. Preston, The algebraic theory of semigroups, Vols. I and II, Math. Surveys of the Amer. Math. Soc. 7 (Providence, R.I., 1961 and 1967).

2. C. Eberhart and J. Selden, One parameter inverse semigroups, Trans. Amer. Math. Soc. 168 (1972), 53-66.

3. D. B. McAlister, Groups, semilattices and inverse semigroups, Trans. Amer. Math. Soc. 192 (1974), 227-244.

4. D. B. McAlister, Groups, semilattices and inverse semigroups II, Trans. Amer. Math. Soc. 196 (1974), 351-369.

5. D. B. McAlister, Some covering and embedding theorems for inverse semigroups, $J$. Austral. Math. Soc. 22 (1976), 188-211.

6. R. McFadden and L. O'Carroll, F-inverse semigroups, Proc. London Math. Soc. (3) 22 (1971), 652-666.

7. W. D. Munn, A class of irreducible matrix representations of an arbitrary inverse semigroup, Proc. Glasgow Math. Assoc. 5 (1961), 41-48. 17-23.

8. L. O'Carroll, A note on free inverse semigroups, Proc. Edinburgh Math. Soc. (2) 19 (1974),

9. L. O'Carroll, Idempotent determined congruences on inverse semigroups, Semigroup Forum 12 (1976), 233-244. 
10. N. R. Reilly and W. D. Munn, E-unitary congruences on inverse semigroups, Glasgow Math. J. 17 (1976), 57-75.

11. N. R. Reilly and H. E. Scheiblich, Congruences on regular semigroups, Pacific J. Math. 23 (1967), 349-360.

Department of Mathematical Sciences

NORTHERN ILLINOIS UNIVERSTTY

DeKalb, IllinoIs 60115, U.S.A. 